TITLE:

\title{
Development and control experiment of the trident snake robot
}

\section{$\operatorname{AUTHOR(S):~}$}

Ishikawa, Masato; Minami, Yuki; Sugie, Toshiharu

\section{CITATION:}

Ishikawa, Masato ...[et al]. Development and control experiment of the trident snake robot. IEEE/ASME Transactions on Mechatronics 2010, 15(1): 9-16: 4797831.

ISSUE DATE:

2010-02

URL:

http://hdl.handle.net/2433/89123

RIGHT:

c 2009 IEEE. 


\title{
Development and Control Experiment of the Trident Snake Robot
}

\author{
Masato Ishikawa, Member, IEEE, Yuki Minami, Student Member, IEEE, and Toshiharu Sugie, Fellow, IEEE
}

\begin{abstract}
This paper is concerned with the development of the trident snake robot, a new example of nonholonomic mobile robot proposed by the authors. The robot has three-pointed shape composed of a center block and three branches, each of which has a passive nonslide wheel. It is modeled as a nonnilpotent driftless system with two generators; its control is a challenging problem, not only because it cannot be treated by continuous control law, but because it cannot be converted to any easy class of nonholonomic systems such as chained form. In this paper, we realized the one-link trident snake robot and applied a periodic control algorithm based on Lie bracket motion. Effectiveness of the proposed algorithm is examined with control experiments.
\end{abstract}

Index Terms-Mobile robot, nonholonomic system, nonlinear system.

\section{INTRODUCTION}

N NAKE robots have been attracting many mechanical and $\checkmark$ control engineers since the pioneering development by Hirose [1], [2]. They are not only stimulating from theoretical viewpoints (especially in terms of nonlinear control) [3], but also useful in practice, such as their hyperredundancy or modularity [4], and mobility in rough terrain with obstacles [5] and underwater environment [6]. Since the snake robots are usually joint-driven with limited angle range, unlike ordinary wheeldriven vehicles, new challenges for actuator technology are also required [7].

In contrast with the biologically inspired snake robots, this paper presents our development and control experiment of the trident snake robot, which is a new kind of wheeled mobile robot proposed in the authors' recent paper [8]. The robot is composed of a center block and three branches of serial links, each of which has the same mechanics as conventional serial snake robots. Each link has a passive wheel rolling on the floor, which causes a nonholonomic constraint. Its kinematic model is described by a driftless nonlinear state equation, classified as nonnilpotent and two-generator systems.

It is impossible to asymptotically stabilize the driftless systems by any continuous state feedback [9], thus many attracting topics have emerged concerning discontinuous or time-varying feedback control design. Among them, single generator systems such as chained systems [10], power systems [11], or

Manuscript received June 9, 2008; revised September 15, 2008. First published March 4, 2009; current version published November 18, 2009. Recommended by Technical Editor J. Ueda.

The authors are with the Department of Systems Science, Graduate School of Informatics, Kyoto University, Kyoto 611-0011, Japan (e-mail: masato@i.kyoto-u.ac.jp).

Color versions of one or more of the figures in this paper are available online at http://ieeexplore.ieee.org.

Digital Object Identifier 10.1109/TMECH.2008.2011985

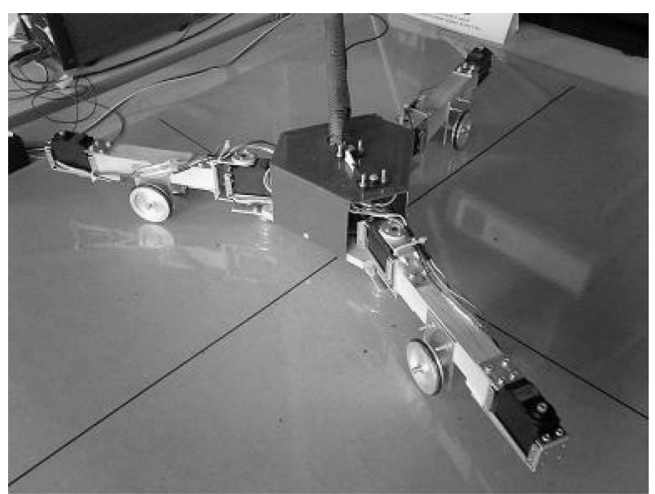

Fig. 1. Trident snake robot.

time-state control systems [12] have been intensively studied since the early 1990s. On the other hand, driftless systems with two or more generators [13] are structurally different from single-generator systems; there have been relatively few attempts [14], [15], and no conclusive control strategy is found up to now. Thus, there are left many challenging problems for nonlinear control theory.

From this point of view, we proposed the trident snake robot as a prototypical example of two-generator system in [8]. There, we analyzed controllability Lie algebra of the system, proposed periodic control algorithms based on the principle of holonomy, and clarified its locomotion principle. We finally succeeded in achievement of physically reasonable motion, up to the level of numerical simulations.

Following these results, this paper aims at realizing the onelink trident snake robot and examining the proposed algorithms in real experiments. One important point to be observed is practical effectiveness of the control algorithm, which is based on the kinematic analysis only. There are several discrepancies between theory and practice such as unmodeled dynamics, backlashes and noises, and selection of feasible periodic trajectories; all of them may cause erroneous result. Thorough the control experiments, we will show that our main purpose of locomotion control is still achievable even with these neglected uncertainties, though the accuracy of control might be affected to some extent.

\section{Kinematic Model of Trident Snake Robot}

Fig. 1 shows an overview of the trident snake robot developed by the authors. Fig. 2 shows its geometric model. In the center of its body, the robot has a root block; an equilateral triangular plate with three actuated joints at its vertexes. Each joint is driven by an actuator. Three links are connected to the root block via the joints, and each link has a passive wheel on its center. 


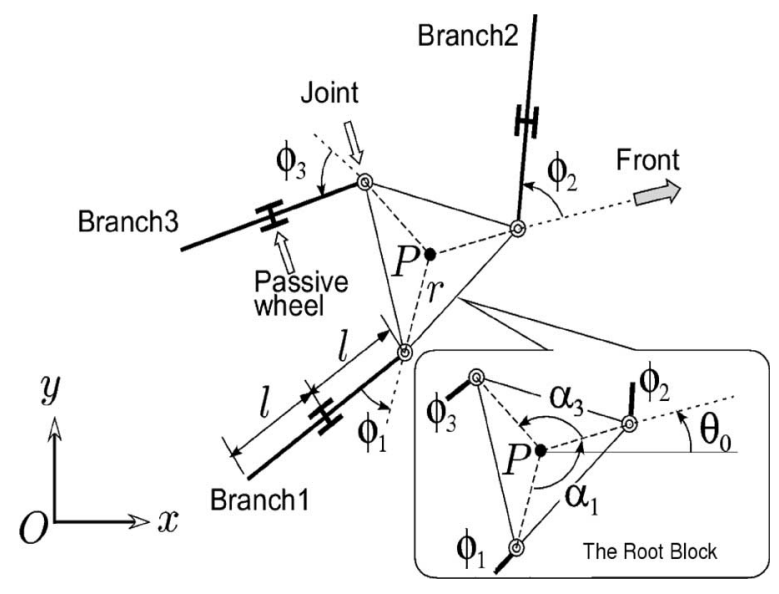

Fig. 2. Trident snake robot (model).

We consider only planar motion in this paper. The robot has a root block with three actuated joints and three branch legs, which are connected to the root block via the joints. Each branch has a passive wheel, which is assumed not to slip, nor slide sideways. In this paper, we assume that model of the robot is the kinematic model and control input is the angular velocity of the joint. Furthermore, we assume that the radius of the triangle is of unit length, and the distance between a joint and a passive wheel is 1 .

The front face of the robot is supposed to be the joint of the second branch $\phi_{2}$. The following constants indicate the directions of the joints $\phi_{i}$ relative to the front face

$$
\alpha_{1}=-2 \pi / 3 \quad \alpha_{2}=0 \quad \alpha_{3}=2 \pi / 3 .
$$

The position of the robot is represented by the coordinates $(x, y)$ of the center $P$. The orientation of the robot is represented by the angle between the $x$-axis and the line passing through $P$ and the joint of branch 2 , say $\theta_{0}$. The configuration vector of the robot is

$$
\boldsymbol{w}:=\left[x, y, \theta_{0}\right]^{T} .
$$

Next, let $\phi_{i}$ denote the joint angle of the $i$ th branch. All the joint variables $\phi_{i}$ are collected in a vector

$$
\phi:=\left[\phi_{1}, \phi_{2}, \phi_{3}\right]^{T}
$$

where $\phi$ is called the shape vector of the robot.

\section{A. Kinematic Model and State Equation}

Now let us turn to the kinematic model of the robot. We assume that the wheels are in ideal rolling contact with the floor, i.e., do not slide nor slip. This assumption leads us to the following nonholonomic constraints

$$
\begin{aligned}
A(\phi) R_{\theta_{0}}^{T} \dot{\boldsymbol{w}} & =B(\boldsymbol{\phi}) \dot{\boldsymbol{\phi}} \\
A(\boldsymbol{\phi}) & =\left[\begin{array}{l}
\sin \left(\alpha_{1}+\phi_{1}\right)-\cos \left(\alpha_{1}+\phi_{1}\right)-l-r \cos \phi_{1} \\
\sin \left(\alpha_{2}+\phi_{2}\right)-\cos \left(\alpha_{2}+\phi_{2}\right)-l-r \cos \phi_{2} \\
\sin \left(\alpha_{3}+\phi_{3}\right)-\cos \left(\alpha_{3}+\phi_{3}\right)-l-r \cos \phi_{3}
\end{array}\right] \\
B(\boldsymbol{\phi}) & =l \boldsymbol{I}_{3}, R_{\theta_{0}}^{T}=\left[\begin{array}{ccc}
\cos \theta_{0} & \sin \theta_{0} & 0 \\
-\sin \theta_{0} & \cos \theta_{0} & 0 \\
0 & 0 & 1
\end{array}\right]
\end{aligned}
$$

where we suppose $r=1, l=1$ for simplicity of notations.

This model has some relevance with a specific type of omnidirectional vehicle with three caster wheels [16], but there is a fundamental difference that the wheels are actively driven in the case of omnidirectional vehicle. If we consider $\boldsymbol{u}:=\dot{\boldsymbol{\phi}}$ is the control input to the kinematic model (2), then we obtain $\dot{\boldsymbol{w}}=R_{\theta_{0}} A(\phi)^{-1} \boldsymbol{u}$. However, for the sake of technical simplicity, we suppress the matrix inverse showing up in the state equation by introducing the following input transformation:

$$
\boldsymbol{v}=A(\phi)^{-1} \boldsymbol{u}
$$

and consider $\boldsymbol{v}$ as a virtual control input from now on.

If $\operatorname{det} A(\phi)=0$, we say the robot is in singular posture. Typical singular postures include the cases in which the nonholonomic constraints degenerate; e.g., two or more wheels are parallel to each other, or all wheels lie on concentric circles. Complete singularity analysis would be a complicated issue; however, the authors checked it numerically and ensured that $\operatorname{det} A(\phi) \neq 0$ holds for $\left|\phi_{i}\right|<\pi / 3$. This suggests that we need not care about the singularity as long as the robot's shape is not too far from $\phi=0$.

Finally, setting $\boldsymbol{\xi}=\left[\begin{array}{c}\phi \\ \boldsymbol{w}\end{array}\right]$ as the state vector, we have the state equation

$$
\dot{\boldsymbol{\xi}}=\left[\begin{array}{c}
A(\boldsymbol{\phi}) \\
R_{\theta_{0}}
\end{array}\right] \boldsymbol{v}=:\left[g_{1}(\boldsymbol{\xi}) g_{2}(\boldsymbol{\xi}) g_{3}(\boldsymbol{\xi})\right] \boldsymbol{v}
$$

where $g_{1}(\boldsymbol{\xi}), g_{2}(\boldsymbol{\xi})$, and $g_{3}(\boldsymbol{\xi})$ are smooth vector fields. Thus, we obtained a three-input and six-state driftless system.

\section{CONTROLlability ANALYSIS AND LOCOMOTION PRINCIPLE}

\section{A. Controllability Analysis}

Let $G$ be a smooth distribution spanned by the input vector fields,

$$
G(\boldsymbol{\xi}):=C^{\infty} \operatorname{span}\left\{g_{1}, g_{2}, g_{3}\right\}(\boldsymbol{\xi}) .
$$

The controllability distribution $\bar{G}$ of the driftless system is defined as the smallest involutive distribution that contains $G$, i.e.

$$
G \subseteq \bar{G} \quad{ }^{\forall} f, g \in \bar{G} \Rightarrow[f, g] \in \bar{G} .
$$

Local controllability of driftless system is completely characterized by the full-rankness of $\bar{G}$ [17].

In the case of the one-link trident snake, $\bar{G}$ is obtained as

$$
\bar{G}(\boldsymbol{\xi})=C^{\infty} \operatorname{span}\left\{g_{1}, g_{2}, g_{3}, g_{12}, g_{23}, g_{31}\right\}(\boldsymbol{\xi})
$$

where $g_{12}:=\left[g_{1}, g_{2}\right], g_{23}:=\left[g_{2}, g_{3}\right]$, and $g_{31}:=\left[g_{3}, g_{1}\right]$. It is easy to see that the system is locally controllable if $A(\phi)$ is 
nonsingular. Indeed, for $\boldsymbol{\phi}=\mathbf{0}$ and arbitrary $\boldsymbol{w}$

$$
\bar{G}(\mathbf{0}, \boldsymbol{w})=\operatorname{span}\left[\begin{array}{cccccc}
-\frac{\sqrt{3}}{2} & \frac{1}{2} & -2 & 1 & -\sqrt{3} & 1 \\
0 & -1 & -2 & 1 & 0 & -2 \\
\frac{\sqrt{3}}{2} & \frac{1}{2} & -2 & 1 & \sqrt{3} & 1 \\
1 & 0 & 0 & 0 & 1 & 0 \\
0 & 1 & 0 & 0 & 0 & 1 \\
0 & 0 & 1 & 0 & 0 & 0
\end{array}\right]
$$

spans the full tangent space $\boldsymbol{R}^{6}$.

Remark 1: From the controllability point of view, the system has the same structure as the so-called first-order systems [18] given by

$$
\dot{\boldsymbol{\xi}}=\left[\begin{array}{ccc}
1 & 0 & 0 \\
0 & 1 & 0 \\
0 & 0 & 1 \\
\xi_{2} & -\xi_{1} & 0 \\
-\xi_{3} & 0 & \xi_{1} \\
0 & \xi_{3} & -\xi_{2}
\end{array}\right] \boldsymbol{v}
$$

Attitude control problem of 3-D spacecraft [19]-[21] is classified in the same class, too. The first-order system is structurally different from the so-called single generator systems such as chained systems [10]; its control is relatively difficult. Moreover, the system (4) is still more difficult to control than (8), because it is not anilpotent system while the latter is nilpotent with the order of nilpotency 2 . It is impossible to convert (4) to (8) exactly, approximate nilpotentization [22] is still possible.

\section{B. Holonomy and Locomotion Principle}

The following fact relates the effect of periodic control and the corresponding Lie brackets, usually known as holonomy or the area rule [17], [23].

Theorem 1: Let $q_{1}(t), q_{2}(t)$ be functions defined on $[0, T]$ and $Q_{1}(t), Q_{2}(t)$ be their integrals

$$
Q_{i}(t):=\int_{0}^{t} q_{i}(\tau) d \tau, \quad i=1,2 .
$$

Moreover, let $q_{1}(t), q_{2}(t)$ be $T$-periodic with mean 0

$$
q_{i}(t+T)=q_{i}(t), \quad Q_{i}(0)=Q_{i}(T)=0, \quad i=1,2 .
$$

Now, suppose that we apply

$$
v_{i}(t)=\epsilon q_{i}(t), \quad \text { for } i=1,2, \quad v_{3}(t) \equiv 0 .
$$

to the driftless system (4) with $\boldsymbol{\xi}(\mathbf{0})=\boldsymbol{\xi}_{0}$ and $\epsilon>0$. Then,

$$
\boldsymbol{\xi}(T)=\boldsymbol{\xi}_{0}+\epsilon^{2} \mathcal{A}\left[g_{1}, g_{2}\right]\left(\boldsymbol{\xi}_{0}\right)+O\left(\epsilon^{3}\right)
$$

holds, where $\mathcal{A}$ is the area encircled by the closed loop on the $Q_{1}-Q_{2}$ plane.

According to this fact, the net change of the state $\boldsymbol{\xi}(T)-\boldsymbol{\xi}_{0}$ under sufficiently small periodic input is approximately equal to $\epsilon^{2} \mathcal{A}\left[g_{1}, g_{2}\right]\left(\boldsymbol{\xi}_{0}\right)$. For instance, consider the following periodic

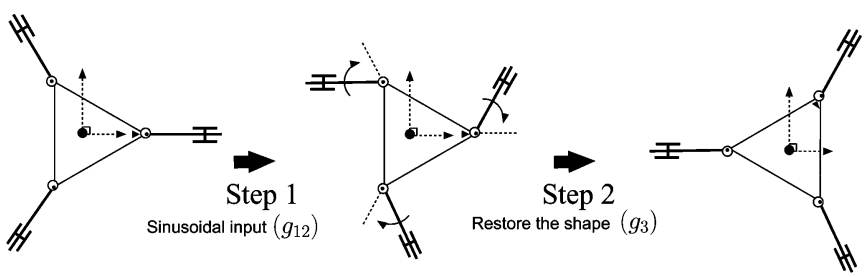

Fig. 3. Rotation control.

input

$$
\boldsymbol{v}(t)=\epsilon[-\omega \sin \omega t, \omega \cos \omega t, 0]^{T}
$$

for $\boldsymbol{\xi}_{0}=\mathbf{0}$ and $T:=2 \pi / \omega$. Then, the resulting change is

$$
\boldsymbol{\xi}(T)=\pi \epsilon^{2}[1,1,1,0,0,0]^{T}+O\left(\epsilon^{3}\right) .
$$

This implies that the joints rotate in counterclockwise (CCW) by the same amount, while the root block comes back to its initial configuration (see Fig. 3).

\section{CONTROL AlgORITHM}

In this section, we briefly describe the rotation and translation control algorithm for the trident snake robot. The previous method proposed in [8] has been improved in some respects to conform to physical experiments. In particular, the problem of shaping periodic trajectory $\left(Q_{1}, Q_{2}\right)$ is worth exploiting; it will be discussed in Sections IV-C and V-E.

\section{A. Rotation Control}

Rotation control is to change the orientation $\theta_{0}$ only, without changing its position $(x, y)$ and shape $\phi$. Without loss of generality, we assume that the initial state is $\boldsymbol{\xi}_{0}=\mathbf{0}$. It is easy to see that a linear combination of $g_{12}$ and $g_{3}$ gives

$$
2 g_{12}(\mathbf{0})+g_{3}(\mathbf{0})=[0,0,0,0,0,1]^{T}
$$

which corresponds to pure rotation. Thus, applying periodic input corresponding to $g_{12}$ followed by constant input corresponding to $g_{3}$ will result in the desired rotation. This idea is summarized by the following algorithm.

\footnotetext{
Algorithm 1. (Rotation control)

For given $\epsilon$ and $\omega$,

Step 1: Apply the periodic input (12) for $t \in[0, T]$ to bring about the displacement (13).

Step 2: Apply constant input $\boldsymbol{v}=[0,0,1]^{T}$ until $\phi$ comes back to $\mathbf{0}$.
}

Continuous rotation is also possible by repeating these two steps. It rotates in clockwise if $\omega$ is set negative $(T:=|2 \pi / \omega|$ in this case).

\section{B. Translation Control}

The purpose of translation control is to change the position of the robot $(x, y)$ in a desired direction by neither changing $\theta_{0}$ nor the joint angles $\phi$. 


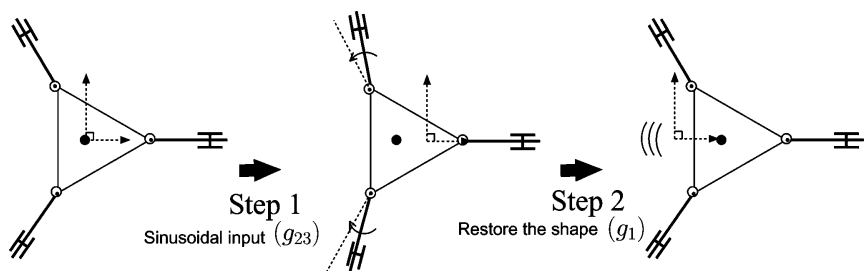

Fig. 4. Translation control $(\beta=0)$.

The control strategy is similar to the previous one. Again, the initial shape is supposed to be $\phi_{0}=\mathbf{0}$. Now let $\beta$ be the angle of the desired direction relative to the $x$-axis and define

$$
\begin{aligned}
& g_{T}:=(\cos \beta) g_{1}+(\sin \beta) g_{2} \\
& g_{N}:=(\sin \beta) g_{1}-(\cos \beta) g_{2} .
\end{aligned}
$$

Now, we find a relation

$$
\left[g_{N}, g_{3}\right](\mathbf{0})+2 g_{T}(\mathbf{0})=[0,0,0, \cos \beta, \sin \beta, 0]^{T} .
$$

This implies that applying periodic input corresponding to $\left[g_{N}, g_{3}\right]$ followed by constant input corresponding to $g_{T}$ will result in pure translation (see Fig. 4). This idea is summarized by the following algorithm.

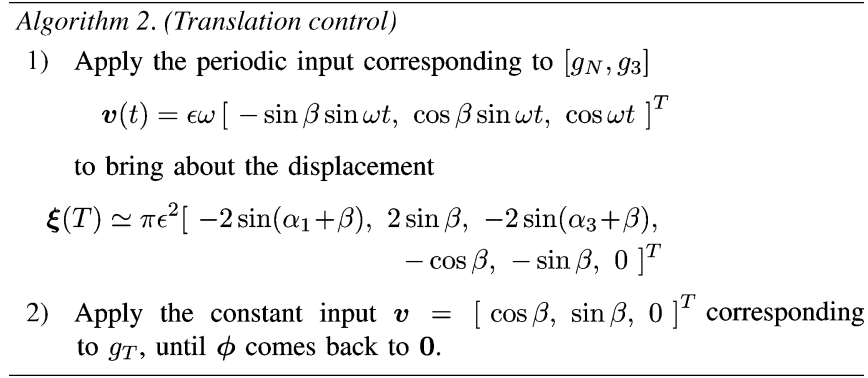

Continuous propulsion is also possible by repeating these two steps.

\section{Selection of Periodic Inputs for Lie Bracket Motion}

Let us briefly discuss the selection of suitable periodic inputs $\left(\epsilon q_{1}(t), \epsilon q_{2}(t)\right)$ in the Step 1 corresponding to the desired Lie bracket.

First, we should note that the approximation (13) is effective as long as $\epsilon$ is sufficiently small. If we set $\epsilon$ too large, the amount of the approximation error increases; namely, the resulting motion is far different from the desired one. On the other hand, the smaller $\epsilon$ is, the slower the robot moves. Thus, moderate $\epsilon$ should be chosen considering this tradeoff.

Second, let us turn to discuss freedom in choosing periodic factors $\left(q_{1}, q_{2}\right)(t)$. There are infinitely many candidates whose integrals $\left(Q_{1}, Q_{2}\right)$ encircle a closed loop having area $\mathcal{A}$. Three of such candidates are shown in Fig. 5 ( $O$ is the origin of $Q_{1}-Q_{2}$ space).

Case (a): This is the simplest case. Just apply

$$
\left(q_{1}, q_{2}\right)=(-\omega \sin (\omega t+\phi), \omega \cos (\omega t+\phi))
$$

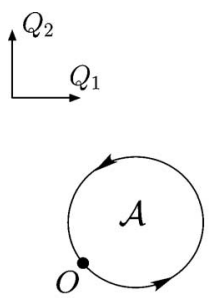

Case (a)

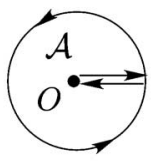

Case (b)

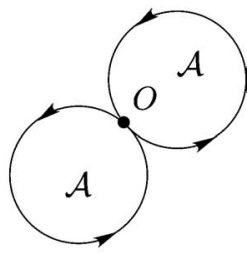

Case (c)
Fig. 5. Possible choices of periodic inputs for Lie bracket motion.

where $\phi \in[0,2 \pi]$ is a constant parameter (initial phase) that determines the center of the circle.

Case $(b)$ : This is slightly modified so that the center of the closed loop coincides with $O$

$\left(q_{1}, q_{2}\right)=\left\{\begin{array}{l}(1,0), \quad t \in[0, \sigma] \\ (-\omega \sin (\omega t-\sigma), \omega \cos (\omega t-\sigma)), \quad t \in[\sigma, \sigma+\mathrm{T}] \\ (-1,0), \quad t \in[\sigma+\mathrm{T}, 2 \sigma+\mathrm{T}] .\end{array}\right.$

In this case, the approximation error is relatively reduced because the maximal distance from $O$ to the points on the curve is smaller than that in case (a).

Case (c): The sign of $\phi$ is flipped alternatively, by combining two periods into a single set

$\left(q_{1}, q_{2}\right)= \begin{cases}(-\omega \sin (\omega t+\phi), \omega \cos (\omega t+\phi)), & t \in[0, T] \\ (-\omega \sin (\omega t-\phi), \omega \cos (\omega t-\phi)), & t \in[T, 2 T] .\end{cases}$

The approximation error in each half period $T$ is as same as in case (a). Since the proposed control algorithms are based on the approximation of the form (13), this approximation error is inevitable. This term results from the infinite series expansion of higher-order Lie brackets, so it is quite difficult to handle it exactly. Nevertheless, it is observed through numerical simulations [8] that the $O\left(\epsilon^{3}\right)$ term is dependent on the choice of the center of the circle. If we trace a pair of circles with antipodal centers as in case (c) of Fig. 5, their $O\left(\epsilon^{3}\right)$ terms would nearly cancel each other and leave smaller error as a result.

This technique is basically a trial to improve the approximation from $O\left(\epsilon^{3}\right)$ to order $O\left(\epsilon^{4}\right)$ by considering the second-order Lie brackets in addition to the first-order one, although its mathematical analysis is still on the way. At this moment, we adopt case (c) as a practical choice in the control experiments. We also compare it with cases (a) and (b) in Section V-E.

\section{EXPERIMENTS}

\section{A. System Setup}

The experimental setup is illustrated in Figs. 6 and 7. Radius of the root block (the distance between the center and a vertex) is $r=0.1 \mathrm{~m}$ and the distance between each joint and wheel is also $l=0.1 \mathrm{~m}$. Total weight of the robot is $1.25 \mathrm{~kg}$ and the size is approximately $540 \mathrm{~mm}$ in diameter.

As for the joint control, we adopted R/C servomotor (Hitec HSR-5995GT; speed $7 \mathrm{rad} / \mathrm{s}$, torque $24 \mathrm{~kg} \cdot \mathrm{cm}$, weight $62 \mathrm{~g}$, and size $40 \mathrm{~mm} \times 20 \mathrm{~mm} \times 37 \mathrm{~mm}$ ) as actuator. This is a compact 


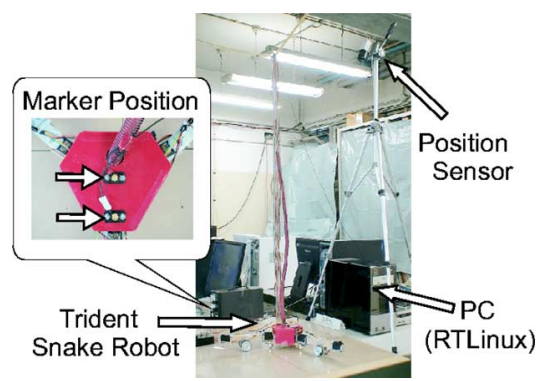

Fig. 6. Experimental setup (overview).

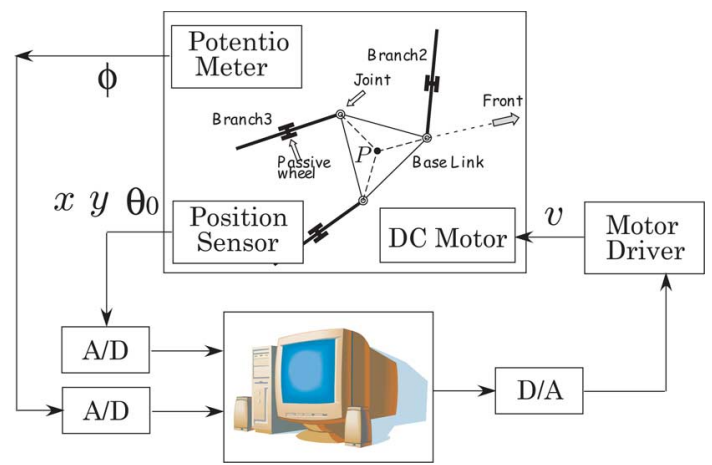

Fig. 7. Experimental setup (component diagram).

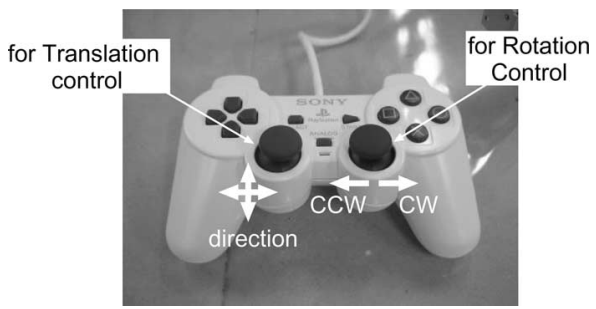

Fig. 8. Manual operation interface.

package including dc motor, gear heads, potentiometer, and peripheral circuits, normally used in radio-controlled vehicles. Joint angle $\phi$ is measured by the potentiometers and sent to the $\mathrm{PC}$ via an $\mathrm{A} / \mathrm{D}$ converter, and the control input is sent to the motor through a digital-to-analog (D/A) converter.

Measurement of the configuration $\boldsymbol{w}=\left(x, y, \theta_{0}\right)$ is often a demanding task in mobile robot control. In this paper, we set up a vision-based measurement system using an image processing system (Hamamatsu Photonics, Position Sensor C5949). A couple of infrared LED markers are fixed on the root block, and detected by a charge-coupled device (CCD) camera fixed overhead. The image processor outputs their positions on the image, from which we can compute their positions on the plane and the configuration $\boldsymbol{w}$, under proper calibration in advance. Control algorithm is performed on a real-time operating system, RTLinux Free, installed in the PC. Additionally, a simple manual operation interface shown in Fig. 8 is also developed so that the operator can send motion commands to the robot. This device enables us to specify the mode (rotation/translation), direction and amplitude of the motion in an interactive way.

\section{B. Tracking Control of Joint Trajectories}

The proposed control algorithms discussed so far were based on kinematics, where the control input $\boldsymbol{u}$ was supposed to be joint angular velocities. In order to realize them with voltageinput dc motors, we introduce simple trajectory tracking control as follows.

Let $\boldsymbol{v}^{*}(t)$ be the control input computed by one of the proposed control algorithms. Recalling the input transformation (3), the reference joint trajectory $\phi^{*}(t)$ is obtained easily by integrating an ordinary differential equation

$$
\dot{\boldsymbol{\phi}}^{*}(t)=A\left(\boldsymbol{\phi}^{*}(t)\right) \boldsymbol{v}^{*}(t) \quad \boldsymbol{\phi}^{*}(0)=\mathbf{0} .
$$

The desired joint angular acceleration $\ddot{\phi}^{*}(t)$ is computed by

$$
\ddot{\boldsymbol{\phi}}^{*}(t)=A\left(\boldsymbol{\phi}^{*}(t)\right) \dot{\boldsymbol{v}}^{*}(t)+\sum_{i=1}^{3} \frac{\partial A}{\partial \phi_{i}}\left(\boldsymbol{\phi}^{*}(t)\right) \dot{\phi}_{i}^{*}(t) \boldsymbol{v}^{*}(t)
$$

or more easily, just by numerical differentiation of $\dot{\phi}^{*}(t)$.

Now, the task has been reduced to make $\phi(t)$ track the reference $\phi^{*}(t)$. Roughly neglecting the interference between joints, dynamics of each joint can be modeled as a linear second-order system

$$
M_{i} \ddot{\phi}_{i}(t)+D_{i} \dot{\phi}_{i}(t)=e_{i}(t), \quad i=1,2,3
$$

where $e_{i}$ is the applied voltage and $M_{i}, D_{i}$ are mass-damper parameters. Consider the following feedback control law:

$$
e_{i}(t)=k_{i}\left(\phi_{i}^{*}(t)-\phi_{i}(t)\right)+M_{i} \ddot{\phi}_{i}^{*}+D_{i} \dot{\phi}_{i}^{*}
$$

where $k_{i}>0$. Then, the tracking error $\bar{\phi}_{i}(t):=\phi_{i}^{*}(t)-\phi_{i}(t)$ obeys the following stable error dynamics:

$$
M_{i} \ddot{\bar{\phi}}_{i}(t)+D_{i} \dot{\bar{\phi}}_{i}(t)+k_{i} \bar{\phi}_{i}(t)=0
$$

implying $\bar{\phi}_{i}(t) \rightarrow 0$.

\section{Rotation Control}

Now, we are ready to examine the proposed control algorithm. Figs. 9-12 show an experimental result of rotation control in $\mathrm{CCW}$, where $\epsilon=0.3$ and $\omega=4.5 \mathrm{rad} / \mathrm{s}$. As mentioned earlier, the periodic pattern (c) in Fig. 5 is used for step 2.

Fig. 9 shows the time response of the joint angles. The solid line shows the actual angle $\phi(t)$ while the dotted one shows the reference $\phi^{*}(t)$. Nice tracking performance to the trajectory is observed despite tininess of the actuators. Note that all the joint angles come back to their initial position $\phi=0$ at the end of step $2(t \simeq 1.8,3.6[s])$.

Fig. 10 shows parametric plot of the position $(x, y)$ on the $x-y$ plane, and Fig. 11 shows the time response of the orientation $\theta_{0}$; the solid lines show the experimental result and the dotted ones show the simulation. Note that the position $(x, y)$ comes back to the origin at the end of step $2(t \simeq 1.8,3.6 s])$, while $\theta_{0}$ increases by about 0.18 [rad] per iteration. Some tracking error in shape control that was not fully compensated is found in Fig. 10. For further improvement of the tracking performance, it is worth trying to redesign the controller parameters $\left(M_{i}, D_{i}, k_{i}\right)$.

Finally, Fig. 12 shows snapshots of an experiment where the algorithm is repeated three times. 


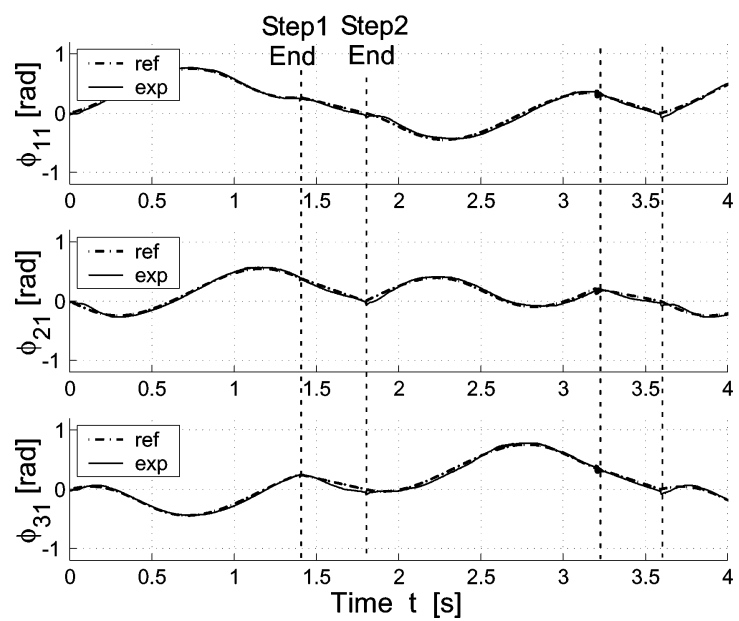

Fig. 9. Time response of joint angles (rotation, $\mathrm{CCW}$ ).

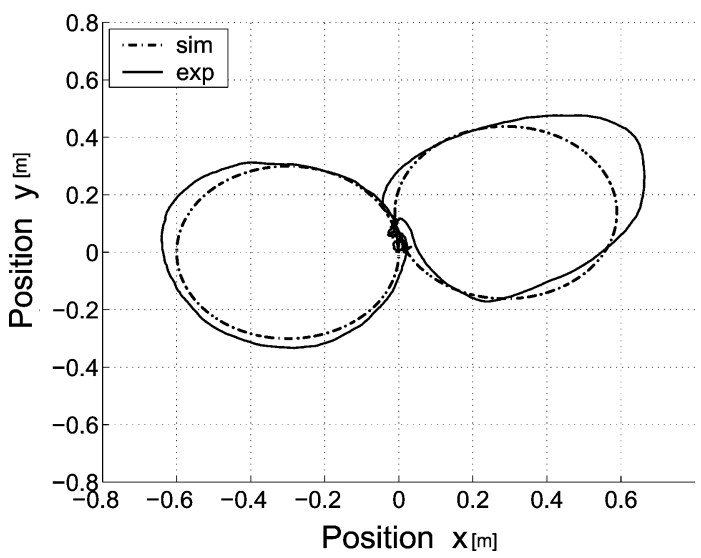

Fig. 10. Parametric plot of position $(x, y)$ (rotation, CCW) (unit added).

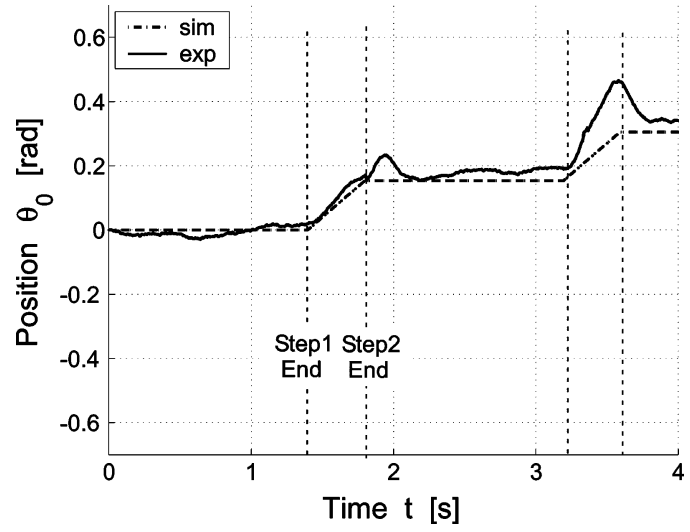

Fig. 11. Time response of $\theta_{0}$ (rotation, CCW).

\section{Translation Control}

Let us turn to an experimental result of translation control along the $x$-axis $(\beta=0)$, where $\epsilon=0.3$ and $\omega=4.0 \mathrm{rad} / \mathrm{s}$. Fig. 13 shows the time response of $x$, which increases by about $3.5 \mathrm{~cm}$ per iteration. Fig. 14 shows snapshots of the result, where the algorithm is repeated three times. We also succeeded in translation control in arbitrary direction specified by $\beta$.

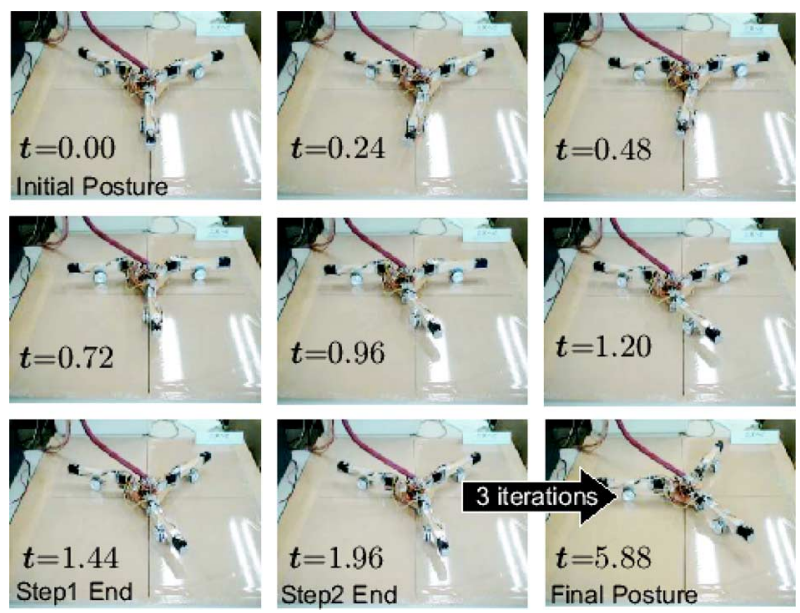

Fig. 12. Snapshots of rotation control.

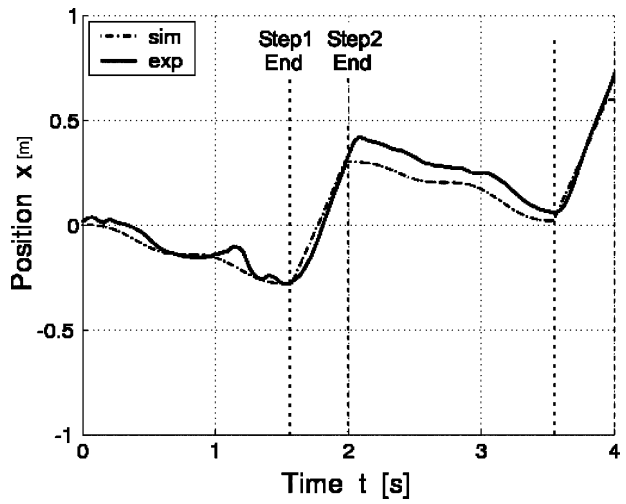

Fig. 13. Result of translation control (along $x$-axis) (unit added).
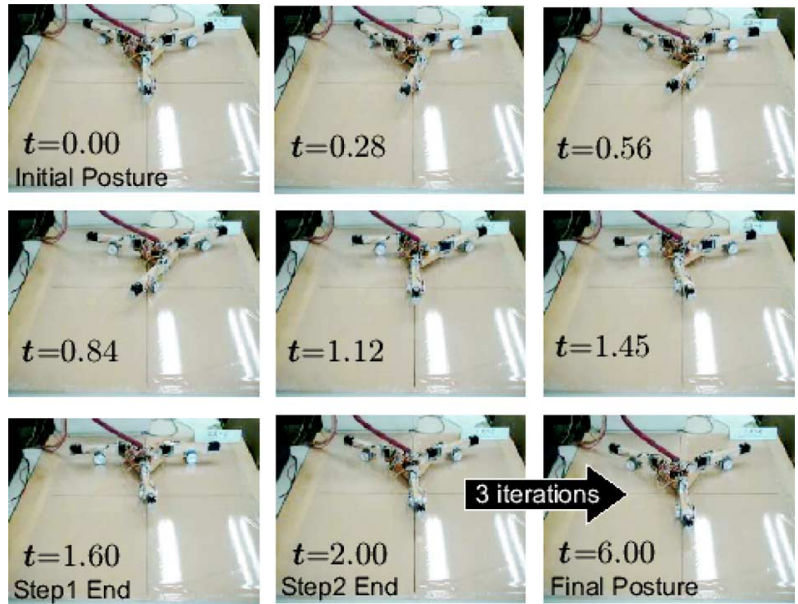

Fig. 14. Snapshots of translation control.

\section{E. Comparison With Cases (a) and (b)}

In this paper, we performed experiments with the periodic input of case (c) in Fig. 5. Let us compare them with the other choices.

Experimental result of rotation control using case (a) is shown in Fig. 15. This is a parametric plot of the position $(x, y)$, where the small dots show the position at the instances of the end of 


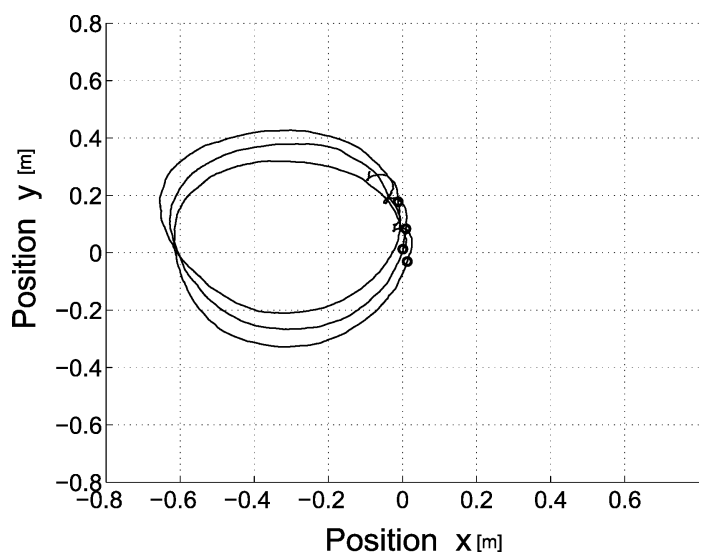

Fig. 15. Experimental result with case (a) (unit added).

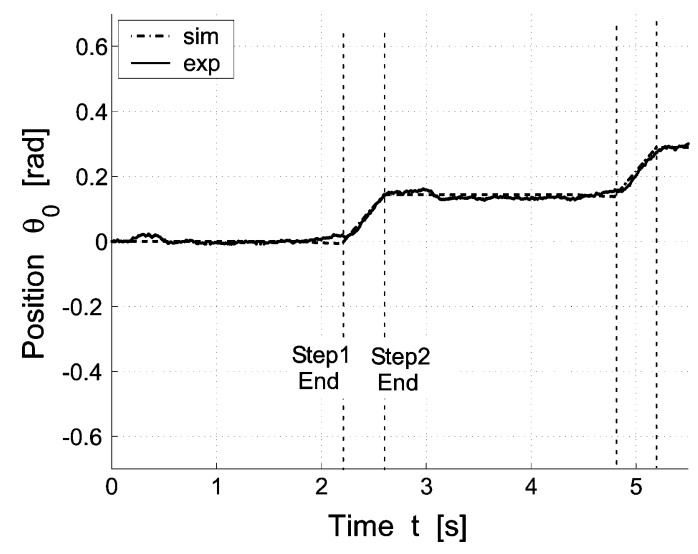

Fig. 16. Experimental result with case (b).

step 1. It is easy to see that the circles drift from the original one as the iteration goes on, due to the approximation error discussed in Section IV-C. This choice is significantly inferior to case (c) on comparing with Fig. 10.

On the other hand, case (b) shows nice performance in Fig. 16 in terms of the time response of the orientation $\theta_{0}$, which is even better than case (c) in Fig. 10. As mentioned in Section IV-C, this is mainly because the position $(x, y)$ is closer to the origin than cases (a) and (c). But the motion of the robot is quicker and smoother in case (c) relative to the speed.

As a consequence, case (c) is comparatively practical for speedy and ceaseless motion, but case (b) may be an alternative choice for slow and precise.

\section{CONCLUSION AND FUTURE WORK}

In this paper, we implemented the one-link trident snake robot and performed physical experiments of rotation and translation control. We confirmed that the proposed algorithm is practical enough with proper choice of periodic input patterns.

The authors expect that this robot will be a common test bed for studying this class of nonholonomic systems (nonnilpotent and two generators). Other approaches, such as discontinuous feedback control [19], switching control [24] or averaging-based control [25], [26] are worth investigating. There remain several tasks to be dealt with in future works. First, the experimental setup needs refinement such as extending the robot to the multilink version and developing the wireless control system with embedded microcomputer. Second, for accurate positioning and/or tracking under the presense of wheel slippage, on-line localization of the robot's position and its feedback is inevitable [27]; the authors are currently working on point-to-point feedback. Other future topics include improvement of the robot's dynamic behavior (16) and intelligent navigation problem including obstacle avoidance [5], [28].

\section{REFERENCES}

[1] Y. Umetani and S. Hirose, "Biomechanical study of serpentine locomotion," in Proc. 1st RoManSy Symp. 1973, pp. 171-184.

[2] S. Hirose, Biologically Inspired Robots: Snake-Like Locomotor and Manipulator. London, U.K.: Oxford Univ. Press, 1993.

[3] J. Ostrowski and J. Burdick, "The geometric mechanics of undulatory robotic locomotion," Int. J. Robot. Res., vol. 17, no. 7, pp. 683-701, 1998.

[4] H. B. Brown, Jr., J. M. Vande Weghe, C. A. Bererton, and P. K. Khosla, "Millibot trains for enhanced mobility," IEEE/ASME Trans. Mechatronics, vol. 7, no. 4, pp. 452-461, Dec. 2002.

[5] A. Transeth, R. Leine, C. Glocker, K. Pettersen, and P. Liljeback, "Online optimization of swimming and crawling in an amphibious snake robot," IEEE Trans. Robot. Autom., vol. 24, no. 1, pp. 88-104, Feb. 2008.

[6] A. Crespi and A. Ijspeert, "Online optimization of swimming and crawling in an amphibious snake robot," IEEE Trans. Robot. Autom., vol. 24, no. 1, pp. 75-87, Feb. 2008.

[7] G. Granosik and J. Borenstein, "Integrated joint actuator for serpentine robots," IEEE/ASME Trans. Mechatronics, vol. 10, no. 5, pp. 473-481, Oct. 2005.

[8] M. Ishikawa, "Trident snake robot: Locomotion analysis and control," in Proc. IFAC NOLCOS, 2004, pp. 1169-1174.

[9] R. Brockett, "Asymptotic stability and feedback stabilization," in Differential Geometric Control Theory, vol. 27, New York: Springer-Verlag, 1983, pp. 181-191.

[10] R. Murray and S. Sastry, "Nonholonomic motion planning: Steering using sinusoids," IEEE Trans. Autom. Control, vol. 38, no. 5, pp. 700-716, May 1993.

[11] J.-B. Pomet and C. Samson, "Time-varying exponential stabilization of nonholonomic systems in power form," INRIA, Sophia Antipolis, France, Res. Rep. 2126, 1993.

[12] M. Sampei, "A control strategy for a class of non-holonomic systemsTime-state control form and its application -," in Proc. 33rd CDC, 1994, pp. $1120-1121$.

[13] M. Ishikawa, "Feedback control of symmetric affine systems with multigenerators," presented at the 15th IFAC World Congr., Barcelona, Spain, 2002.

[14] A. Agrachev and Y. Sachkov, "Optimal control for a nonlinear driftless 5-dimensional system with 2 inputs," in Proc. 5th IFAC Symp. Nonlinear Control Syst. (NOLCOS 2001), pp. 60-63.

[15] J.-P. Gauthier and V. Zakalyukin, "On the one-step-bracket-generating motion planning problem," J. Dyn. Control Syst., vol. 11, no. 2, pp. 215235,2005

[16] G. Campion, G. Bastin, and B. D’Andrea-Novel, "Structural properties and classification of kinematic and dynamic models of wheeled mobile robot," IEEE Trans. Robot. Autom., vol. 12, no. 1, pp. 47-62, Feb. 1996.

[17] S. Sastry, Nonlinear Systems-Analysis, Stability, and Control. New York: Springer-Verlag, 1999.

[18] R. Murray, Z. Li, and S. Sastry, A Mathematical Introduction to Robotic Manipulation. Boca Raton, FL: CRC Press, 1994.

[19] T. Tsuchiya, T. Urakubo, and K. Tsujita, "Motion control of a nonholonomic system based on the Lyapunov control method," J. Guid., Control, Dyn., vol. 25, no. 2, pp. 285-290, 2002.

[20] N. Sreenath, "Nonlinear control of planar multibody systems in shape space," Math. Control, Signals, Syst., vol. 5, no. 5, pp. 343-363, 1992.

[21] A. Guichardet, "On rotation and vibration motions of molecules," Ann. Inst. Henri Poincare, vol. 40, no. 3, pp. 329-342, 1984.

[22] H. Hermes, "Nilpotent and high order approximations of vector fields systems," SIAM Rev., vol. 33, pp. 238-264, 1991. 
[23] N. Leonard and P. Krishnaprasad, "Motion control of drift-free, leftinvariant systems on lie groups," IEEE Trans. Autom. Control, vol. 40, no. 9, pp. 1539-1554, Sep. 1995.

[24] Y. Iwatani, M. Ishikawa, and S. Hara, "Switched feedback control of firstorder symmetric affine systems via time-state control form," in Proc. ACC 2002 , pp. 2516-2521.

[25] F. Bullo, N. Leonard, and A. Lewis, "Controllability and motion algorithms for underactuated lagrangian systems on lie groups," IEEE Trans. Autom. Control, vol. 45, no. 8, pp. 1437-1454, Aug. 2000.

[26] F. Bullo, "Averaging and vibrational control of mechanical systems," SIAM J. Control Optim., vol. 41, no. 2, pp. 542-562, 2002.

[27] C. B. Low and D. Wang, "Gps-based tracking control for a car-like wheeled mobile robot with skidding and slipping," IEEE/ASME Trans. Mechatronics, vol. 13, no. 4, pp. 480-484, Aug. 2008.

[28] K. Jan, G. Y. Chang, and I. Parberry, "Optimal path planning for mobile robot navigation," IEEE/ASME Trans. Mechatronics, vol. 13, no. 4, pp. 451-460, Aug. 2008.

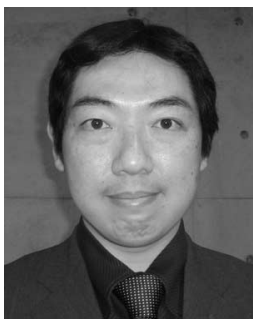

Masato Ishikawa (S'94-M'00) received the B.E., M.E., and Ph.D. degrees in engineering from Tokyo Institute of Technology, Tokyo, Japan, in 1994, 1996, and 2000, respectively.

From 1999 to 2002, he was a Research Associate at Tokyo Institute of Technology. From 2002 to 2004, he was a Research Associate at the University of Tokyo, Tokyo. In 2004, he joined Kyoto University, Kyoto, Japan, where he is currently a Senior Lecturer in the Department of Systems Science. His current research interests include nonlinear control, nonholonomic mechanics, and their application to robotic systems.

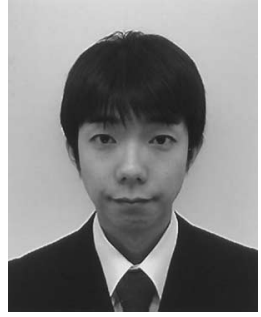

Yuki Minami (S'08) received the M.S. degree in informatics in 2007 from Kyoto University, Kyoto, Japan, where he is currently working toward the Ph.D. degree.

$\mathrm{He}$ is currently a Research Fellow of the Japan Society for the Promotion of Science. His current research interests include quantized control and control of mechanical systems.

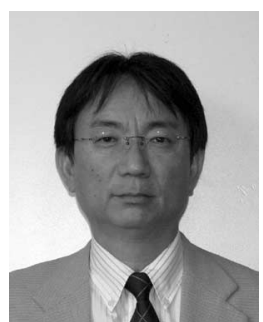

Toshiharu Sugie (M'89-SM'02-F'07) received the B.E., M.E., and Ph.D. degrees in engineering from Kyoto University, Kyoto, Japan, in 1976, 1978, and 1985 , respectively.

From 1978 to 1980, he was a Research Member with the Musashino Electric Communication Laboratory, NTT, Musashino, Japan. From 1984 to 1988, he was a Research Associate in the Department of Mechanical Engineering, University of Osaka Prefecture, Osaka, Japan. In 1988, he joined Kyoto University, where he is currently a Professor in the Department of Systems Science. His current research interests include robust control, learning control, nonlinear control, identification for control, and their application to mechanical systems. 